\title{
From Molecular Hollows to Imprinting Strategies - Mass-Sensitive Devices in Host-Guest Chemistry
}

\author{
Usman Latif $^{1}$, Franz L. Dickert ${ }^{2 *}$ \\ 1 COMSATS Institute of Information Technology, Department of Chemistry, Tobe Camp, University \\ Road, 22060 Abbottabad, Pakistan \\ 2 Department of Analytical Chemistry, University of Vienna, Waehringer Strasse 38, A-1090 Vienna, \\ Austria
}

* Author to whom correspondence should be addressed; E-Mail: Franz.Dickert@univie.ac.at

Received: 03 September 2012/ Accepted: O1 October 2012 / Published: 08 November 2012

\begin{abstract}
Chemically sensitive coatings via supra-molecular chemistry are preferably combined with mass-sensitive devices. Thus, the quartz-crystal-balance (QCM) and the surface acoustic wave (SAW) oscillator allow monitoring inclusion phenomena from nanogram down to the sub-femto-gram range. A deeper insight into the inclusion process of analytes by e.g. cyclodextrines, calixarenes and paracyclophanes is obtained by modelling procedures, which offer to predict the sensitivity and selectivity of sensor coatings. The robustness of the materials is further enhanced by cross-linking which leads to functionalized polymers. This strategy is further developed by templating, in most cases identical to analytes, the polymer materials enable us to perform both gas- and liquid-phase measurements due to their inertness. The flexibility of imprinting technique allows us to produce more selective and optimized recognition sites for the detection of aromatic, halogenated, polycyclic hydrocarbons and even complex mixtures e.g. oil degradation, composting are also accessible to monitor at isomeric level.
\end{abstract}

Keywords: chemical sensors; supramolecular chemistry; solvent vapors; polyaromatic hydrocarbons; engine oil; composting. 


\section{Introduction:}

Chemical sensors are miniaturized, low cost devices and can be tuned for a number of applications by combining a chemically sensitive layer with mass-sensitive transducers (1-3). These devices can offer a very cheap and reliable way for real time analysis of different processes without complicated sampling. A sensitive coating should possess interaction sites, which can incorporate analyte molecules selectively and reversibly $(4,5)$. Supramolecular chemistry helps us to develop a sensor layer by utilizing such compounds which have molecular cavities and are capable to accommodate targeted molecules via host-guest mechanism (6-9). Thus, cyclodextrines, paracyclophanes, and calixarenes are utilized as host molecules because the size of hollows in these structures can be customized for targeted guests by varying their subgroups. The ring like structure of cyclodextrines (10), originate from variable number of glucose molecules, exhibit hydrophobic properties to incorporate guests. Their capability can be increased further by integration with polymeric structures through hydroxyl groups. The nitrogen in paracyclophane (11) can act as interaction centre via selective hydrogen bonding with guest molecules. The molecular flexibility of calixarenes via conformational changes in structure makes them an outstanding receptor for number of guest molecules (12-14). The cross sensitivity of calixarenes and cyclodextrines towards humidity can be decreased by silylation or methylation, to enhance their hydrophobic character, while using their sensor coatings in gas phase measurements (15). In liquid phase measurements, calixarenes are tuned for inclusion of ionic species and cyclodextrines for catalyzing hydrophobic reactants (16). The supramolecular cavities in these sensitive coatings offer considerable interactions towards different analytes (17) but a more detailed understanding of incorporation behavior can be visualized as three dimensional structures of the host-guest complexes by chemical modeling based on force field calculations (18-20). These modeling packages also allow us to predict the interaction energies for the inclusion of analytes. The pre-organized molecular hollows as sensitive coatings in combination with a transducer can be applied for wide field of applications. Another flexible method for generating more selective interaction sites in sensor materials is molecular imprinting (21-23). A highly cross-linked porous matrix is synthesized by polymerizing different functional monomers along with template molecule. Self-organization of these monomers engulfs the guest molecule during polymerization. The template can be removed by heating or washing with appropriated solvent and this process will originate active sites in polymer material (24). The choice of the model compound depends on cavities to be generated. These imprinted materials offer stereo chemically fit recognition sites in the whole polymeric configuration, for the selective inclusion of analytes such as the antibody and antigen interactions in biological systems (25). The imprinting technique is more flexible in generating recognition systems which makes them superior to molecular hollows, based on supramolecular chemistry, in terms of stability, ease of preparation and most importantly cost (26).

\section{Results and Discussion:}

\subsection{Molecular cavities}

\subsubsection{Cyclodextrins}

In host-guest chemistry the recognition of the targeted guest can be successfully achieved by incorporation into the cavity of the host and not being absorbed outside, which leads to the high 
selectivity of the material. By following this strategy, the sensor materials can be designed for the detection of number of analytes with the variation of cavity diameter. Thus, the response of gravimetric sensor directly depends on the molecular hollows in the sensitive layer. Here in this case, the cyclodextrins are employed as host because they form a cavity of variable number of glucose molecules, which encircle the guest molecules, as depicted from the sensor effects - relative frequency changes increase in parallel to perchloroethylene concentration. The shape of cyclodextrin ring is like a cone, while, the hydroxyl groups of glucose situated outside. Therefore, host molecules show hydrophobic properties from inside and leads to the incorporation of targeted guest of suitable polarity, and by modeling we have found that glycoside is no efficient donor to e.g. acidic-H. The presence of hydroxyl groups outside the cone exhibits strong interactions with water vapors. This cross sensitivity to water can be strongly reduced during gas-phase detection of solvent vapors if hydroxyl groups are silylated or methylated (27). Permethylated $\alpha$ - and $\beta$-cyclodextrins are used as sensor material for the detection of perchloroethylene as shown in figure 1 . This analyte is chosen on the basis of its economic relevance, as it is being used in dry cleaning. The measurements are performed by coating the sensor materials on one electrode of dual electrode QCM (10 MHz), while, second electrode serves as reference. An appreciable sensor response is observed for $\beta$-cyclodextrin and $\alpha$-cyclodextrin shows negligible effects against solvent vapors. From these measurements, we can conclude that cavities of $\beta$-cyclodextrin are perfectly fit for solvent molecules in spite of $\alpha$-cyclodextrin, which are too small and the recognition of perchloroethylene is actually achieved by the incorporation of solvent vapors into the cavities.

Figure 1. Mass sensitive responses to perchloroethylene while coating the transducer with methylated $\alpha$ - and $\beta$-cyclodextrins of thickness 100nm.

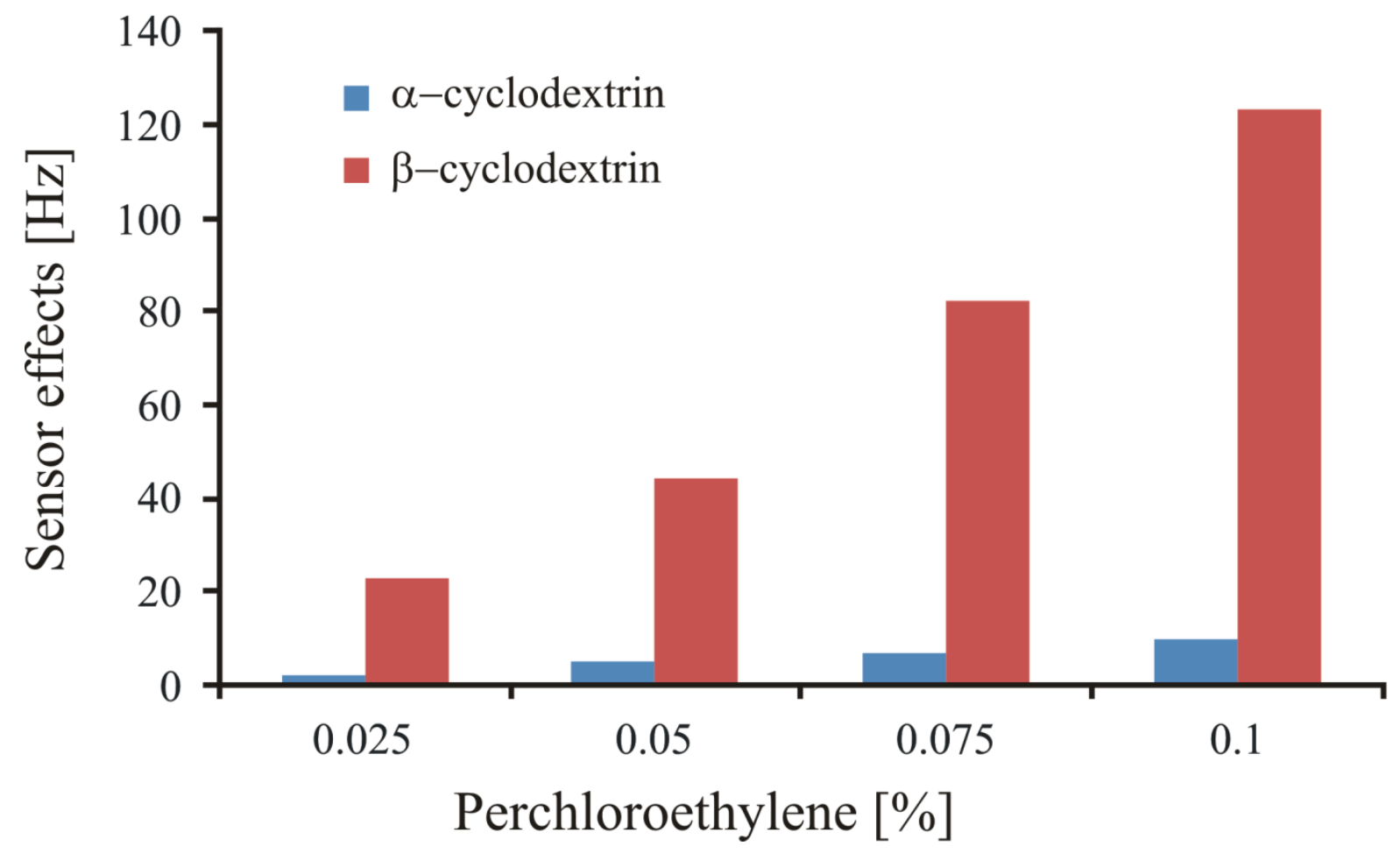


If the recognition takes place outside the molecular hollows, then, both sensors should show equal effects. $\alpha$-, $\beta$-, and $\gamma$-cyclodextrins are originated from 6,7 , and 8 glucose units and having cavities with diameter of 4.7-5.2 $\AA$, 6.0-6.5 $\AA$, and 7.5-8.5 $\AA$, respectively. The diameter of $\beta$-cyclodextrin cavities is approximately similar to perchloroethylene $(6.5 \AA, \mathrm{Cl}-\mathrm{C}-\mathrm{Cl})$, which, leads to the appreciable sensor response in comparison to $\alpha$-cyclodextrin as sensitive layer. The response time of these materials are approximately few seconds and their stability is of more than two years. Some aromatic vapors are also exposed to silylated and non-silylated $\beta$-cyclodextrine and their respective responses are shown in figure 2.

Figure 2. Comparison of silylated and non-silylated cyclodextrins towards some aromatic solvents effects of substituents on selectivity.

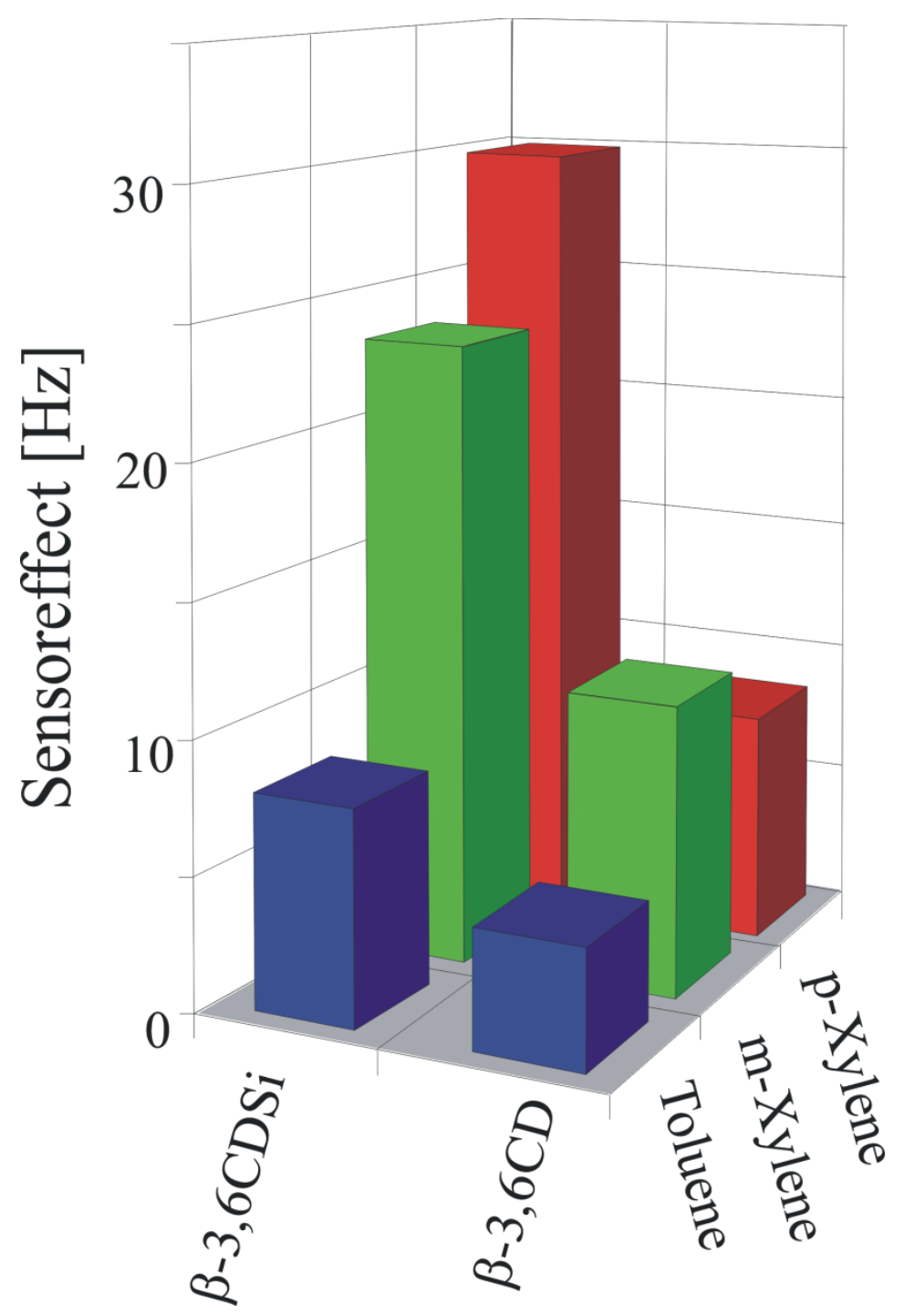

The sensor effects of $\mathrm{m}$ - and $\mathrm{p}$-xylene are different to silylated and non-silylated coatings. This behavior of cyclodextrins can be explained in such a way that silylation leads to the slightly elongated cone of host molecule and offers a suitable cavity to p-xylene, whereas, m-xylene is more bulky and steric hindrance caused by methoxy group suppress its accessibility to available cavities in comparison to the non-silylated sugars. The variation of silylation allows us to tune the sensitivity of the cyclodextrins in such a way that meta- and para-isomers can be differentiated. 
Sensitive layer of $\beta$-cyclodextrin is coated on quartz crystal microbalance (10MHz) and exposed to different solvent vapors $(0.5 \%$ in the air), as shown in figure 3 . Molecular modeling helps us to understand recognition capability of host molecules. The results of MM3 calculations $\left(\Delta \mathrm{G}^{\mathrm{o}}\right)$ of the respective host-guest complexes are plotted against $\mathrm{lnK}$ which is directly calculated from sensor responses. The sensor responses are attributed to the formation of host-guest complexes and equilibrium constant $\mathrm{K}$ of this process is calculated from the corresponding sensor signals. Gibbs energies $\Delta \mathrm{G}^{\mathrm{o}}$ of host-guest complex formation is related to equilibrium constant $\mathrm{K}$ by $\Delta \mathrm{G}^{\mathrm{o}}=-\mathrm{RT} \ln \mathrm{K}$. These Gibbs energies $\Delta \mathrm{G}^{\mathrm{o}}$ are approximated by using MM3 force field calculations and are directly related to the equilibrium constant $\mathrm{K}$ (28). The results exhibit the behavior of cyclodextrin hosts towards different solvent vapors. The introduction of guest molecules such as xylene increases the interaction behavior due to the better morphological fit in the cavities with increased surface area and this interaction decreases by exposing to toluene and benzene vapors. The cyclodextrin pockets do not offer an effective interaction to chlorinated hydrocarbons of smaller size such as carbon tetrachloride and trichloromethane in comparison to the tetrachloroethylene. These results also prove that cavities in host material are big enough for binding to organic solvent molecules.

Figure 3. Correlation between the equilibrium constant $K$, derived from sensor effects of $\beta$ cyclodextrin ( $\beta-C D)$, modified by a cap of hexa fluorobenzene (HFB), to different solvent vapors $\left(0.5 \%\right.$ in the air, per: tetrachloroethylene) and the Gibbs energies $\left(\Delta \mathrm{G}^{\mathrm{o}}\right)$ of the respective host-guest complexes approximated by MM3 calculations.

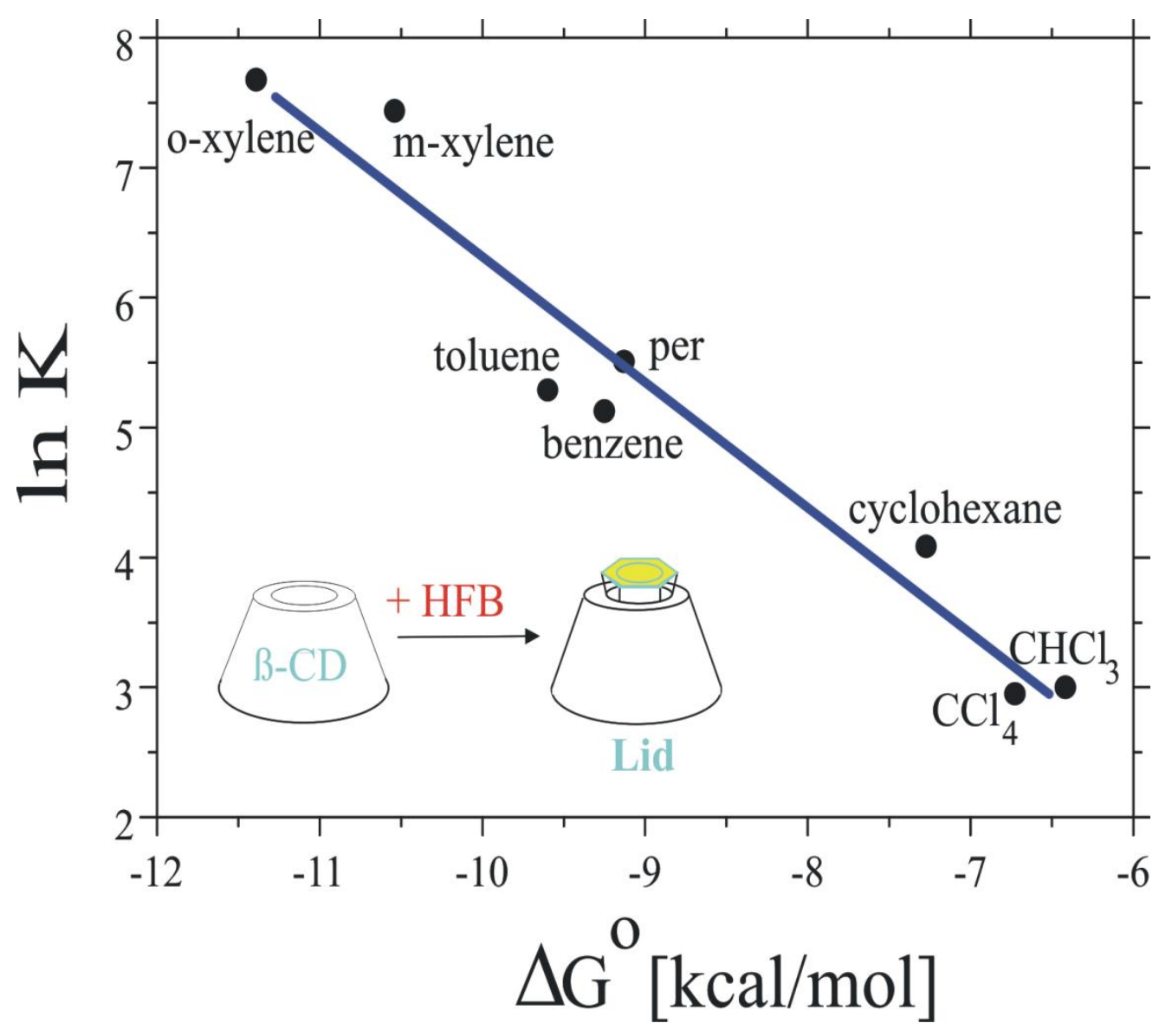




\subsubsection{Paracyclophanes}

Paracyclophanes can also be used as host for the inclusion of guest molecules due to the cavities present in their structures. Tetraazaparacyclophanes are used as sensitive coatings for gravimetric sensors. These coatings offer somewhat elongated cavity which is best for flat molecules. The geometry of cavity in cyclophanes can be adjusted by varying the aliphatic spacer groups, which allow the adaption to size of molecule. In order to increase the oxidation stability and reduction of water cross sensitivity, the amino bridgeheads are converted to tertiary amino functions by introducing aliphatic substituents. Sensitive layer of $N, N^{\prime}, N^{\prime \prime}, N^{\prime \prime \prime}$-tetra(n-pentyl)-1,6,20,25-tetraaza-13,32dioxo[6.1.6.1] paracyclophane (CPO44VAL) is coated on SAW (433 $\mathrm{MHz}$ ) and exposed to tetrachloroethylene $(0.5 \%$ in the air), as shown in figure 4 . The diphenylether pockets offer an effective interaction to chlorinated hydrocarbons due to their high polarizability. The result also proves that cavities in host material are big enough for binding to tetrachloroethylene. Fast response time, better sensitivity, and reversibility are observed in comparison to QCM experiment with a typical $t_{90}$ time of about three to five minutes.

Figure 4. Sensor effect to an increasing concentration of tetrachloroethylene while coating SAW device with CPO44VAL followed by analyte free air.

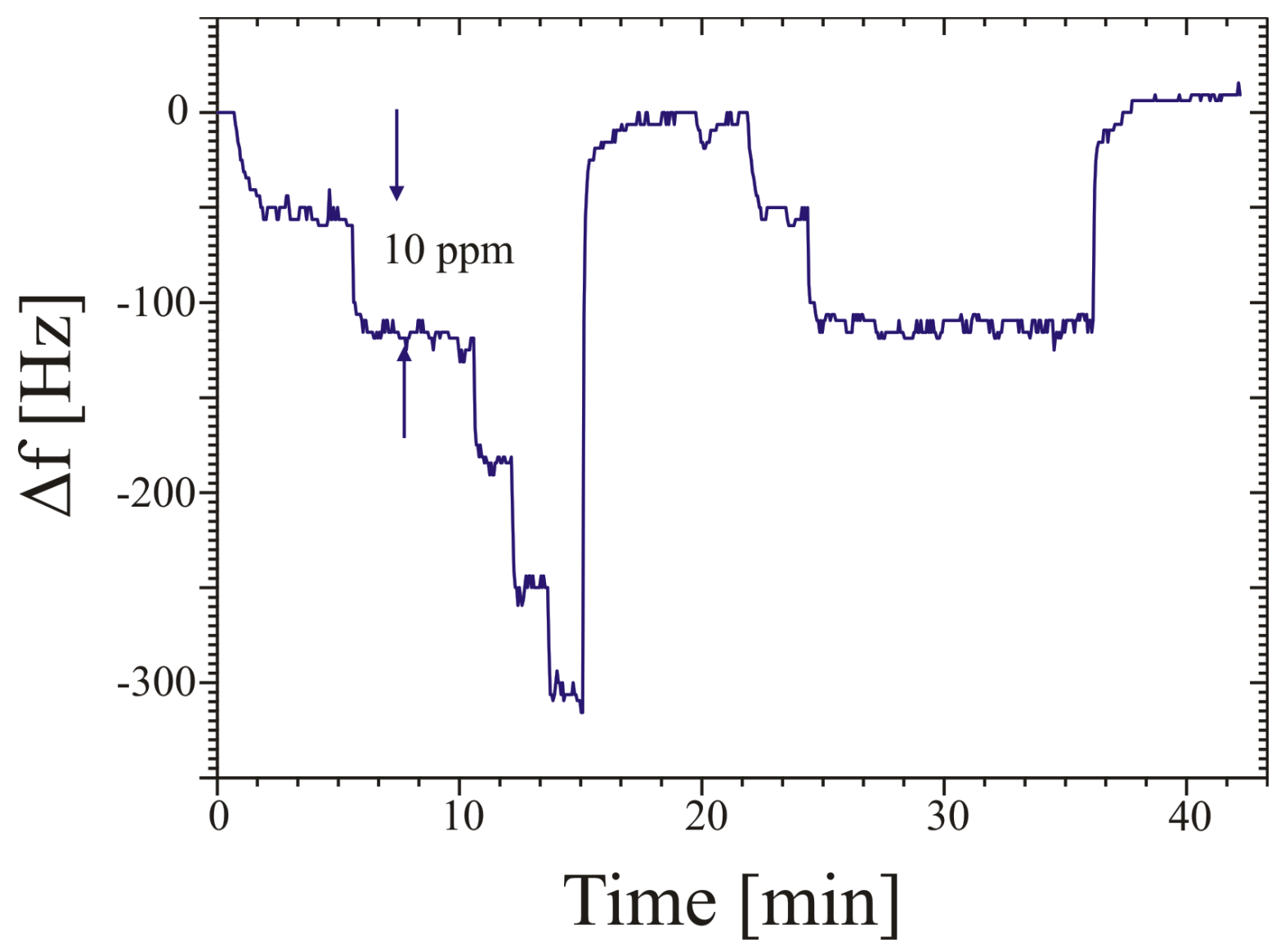

\subsubsection{Calixarenes}

Calixarenes as host offers high molecular flexibility and better adaptation to the analytes by an induced conformational change, which makes them an outstanding receptor for a number of guest molecules. Thus, these host molecules can be tuned for number of application i.e. for solvent vapor 
recognition in gas phase or inclusion of ionic species in liquid phase. In calix $[n]$ arenes $(n=4,6,8)$ the rotational flexibility of phenyl groups around single bond allows them to exist in different conformations. Therefore, tert-butyl-calix[4]arenes exhibit four different conformations such as "cone", "partial cone", "1,2-alternate", "1,3-alternate". Tert-butyl-calix[6]arenes favors two out of eight conformers, which are termed as "hinged" or "winged". In "hinged" conformation three contiguous phenyl groups are up and other three are down, while, in other conformation, two opposite aryl groups are in up and other four in down positions and form an easy accessible cavity.

The tert-butyl-calix[8]arenes are very large and rotational flexibility of phenyl groups around single bond allows them to form 16 different conformers. Out of which, "hinged" conformations is favorable having four rings are up and other in down positions building a double-cone structure available to accommodate two guest in one host molecule. This conformation as sensitive coatings leads to a very high sensor response. All hydroxyl groups in tert-butyl-calix[n]arenes are modified with methyl and trimethylsilyl groups in order to synthesize hydrophobic coatings with negligible water crosssensitivity. The silylated calix[8]arene as sensitive coating on $433 \mathrm{MHz}$ SAW device allows us to measure the increasing concentration of tetrachloroethylene, as shown in figure 5. This is due to the fact that phenyl rings offer excellent electronic interactions by their polarizability. This effect is more pronounced in calixarenes compared to cyclodextrins.

Figure 5. Sensor response of silylated tert-butylcalix[8]arene for tetrachloroethylene on $433 \mathrm{MHz}$

SAW oscillator.

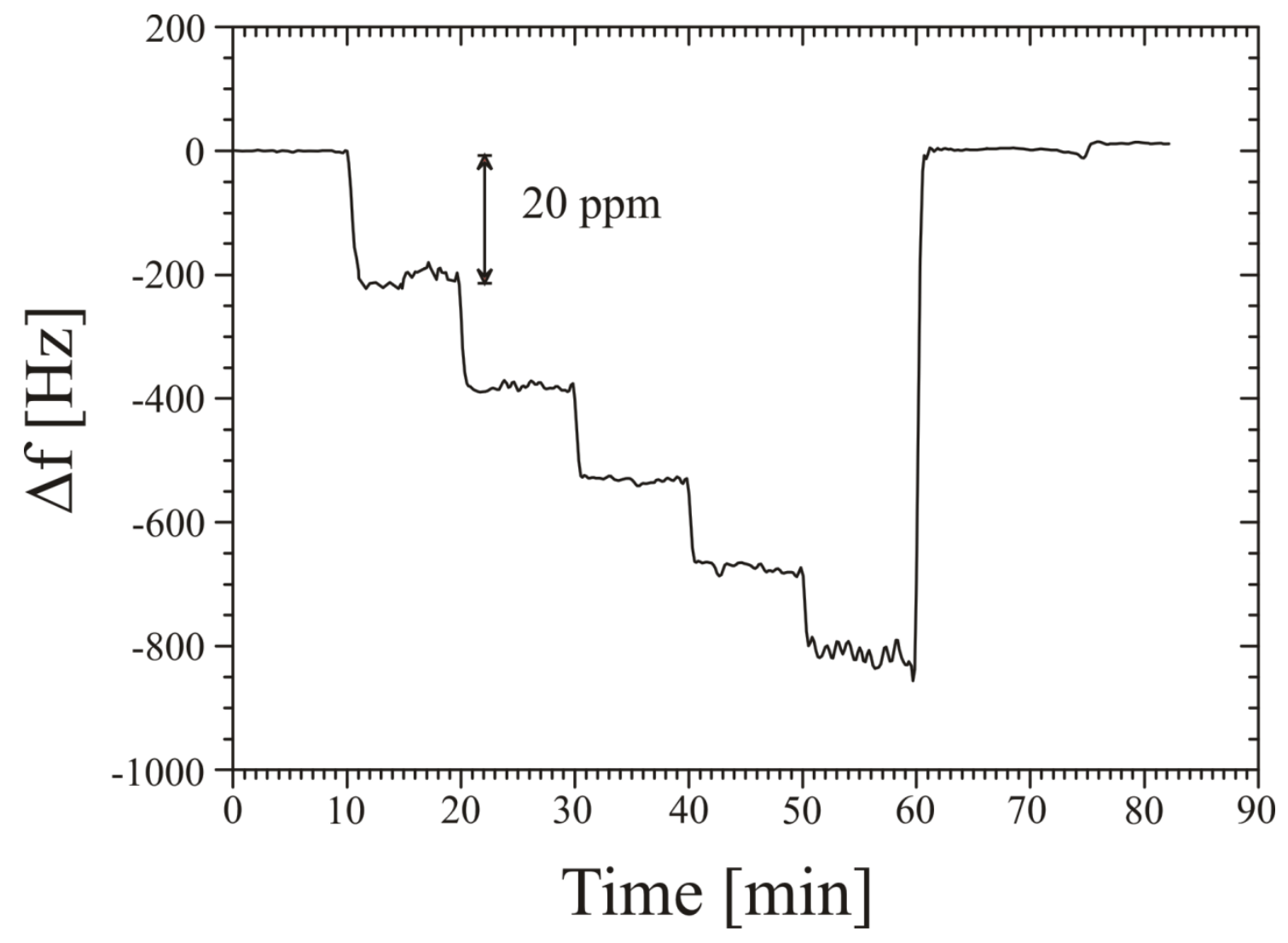




\subsection{Molecular imprinting}

\subsubsection{Molecular imprinted PAH sensor}

Molecular imprinting is a very straightforward and universally adaptive technique to design a recognition pattern in materials. To produce a desired structure in materials, a template is added along with the monomers during synthesis. The template may be targeted analyte or any suitable compound can be inserted, which does not react with the monomers during polymerization. This polymeric material engulfs the template and afterwards, removal of this model compound from polymer produce recognition sites similar to the template. The accessibility, interactions with targeted analyte (rebinding), to these complementary sites is through the pores (diffusion channels) of polymeric structure developed during polymerization. The structuring of polymers in such a way originates outstanding sensitive materials which offer reversible and selective inclusion of targeted analytes. These rigid molecular imprinted layers are suitable for the detection of analytes both in liquid and gaseous phase due to their inertness against water and organic solvents. The incorporation of analytes in available polymeric hollows depends on their sizes of cavities and diffusion pathways. If the cavity is too small then, it will not accommodate the incoming analytes and if it's too wide then analytes can be easily extracted and layers would not be enriched with analytes. Moreover, the suitable diffusion channels make the recognition sites easily accessible for incoming compounds. Thus, the selectivity pattern of these imprinted polymers depends on template molecule, porogen (in order to generate highly porous structure), and polymerizing conditions. Sometimes, unselective condensations occur during polymerization which leads to narrowing the diffusion pathways, thus, block the accessibility to the recognition sites (29).

An imprinted polyurethane layer is generated by templating with anthracene followed by investigating its enrichment factor and sensor effects towards chrysene and perylene. The sensor responses are measured by coating anthracene-imprinted layer on one channel of dual-electrode 10$\mathrm{MHz} \mathrm{QCM}$, and the second channel is covered with non-imprinted polyurethane layer to cancel temperature and pressure fluctuations. The mass-sensitive sensor is exposed to $2 \mu \mathrm{gL}^{-1}$ concentrations of chrysene and perylene in water, respectively.

The sensitive layer exhibits higher sensor effects towards chrysene. The template must not necessarily be identical to the analyte. A similar sensitivity pattern can also be observed while investigating the fluorescence intensities of chrysene and perylene, as shown in figure 6. Thus, we can say that the hollows in sensitive coatings are optimized in size and shape, which can offer geometrically fit recognition sites and diffusion channels for incoming analytes, as confirmed by masssensitive and fluorescence measurements. 
Figure 6. Mass-sensitive and fluorescence sensor response of anthracene-imprinted polyurethane layer to chrysene and perylene.

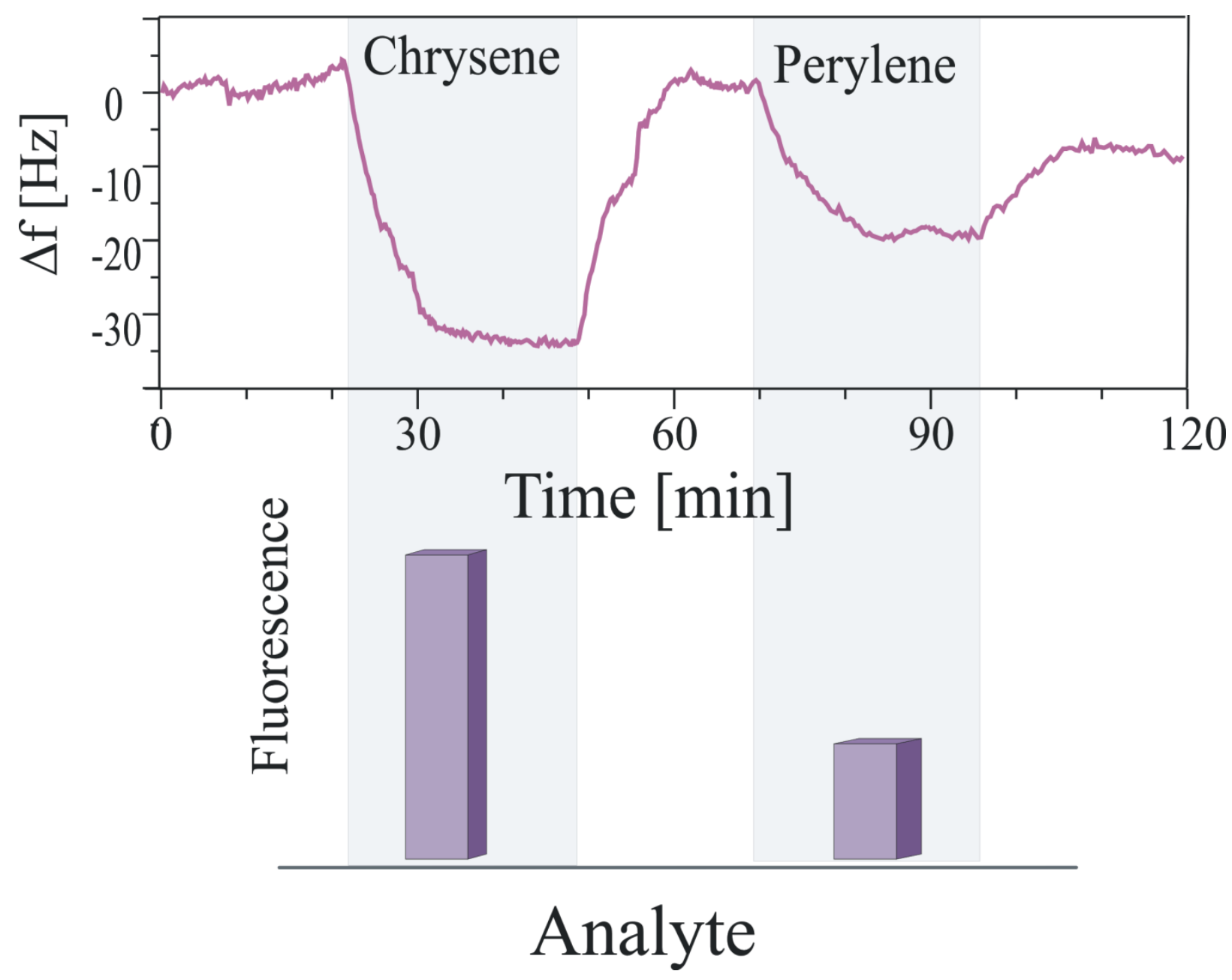

The material properties also play a key role in developing the interactions between recognition sites and analytes. In this way, the hydrophobic and hydrophilic properties of polyurethane layer are important for the inclusion of molecules. The saturation is investigated by UV and fluorescence measurements with a detection limit of $30 \mathrm{ng} / \mathrm{L}$ and response time of few minutes. A linear relationship between signal intensity and layer height approves that saturation is a bulk effect. Figure 7 exhibit the influence of polymerization temperature on the sensitivity pattern of double imprinted polyurethane layers towards anthracene. Perylene and naphthalene are combined in different ratios for printing process. At $20^{\circ} \mathrm{C}$ and $70^{\circ} \mathrm{C}$, the pronounced enrichment is observed with $25: 75$ or $75: 25$ of perylene and naphthalene. However, maximum inclusion of anthracene in recognition sites is obtained at $45^{\circ} \mathrm{C}$ with 50:50\% of perylene and naphthalene. This result indicates that the polymer matrix is more closely arranged around template during polymerization at $45^{\circ} \mathrm{C}$ in order to generate the cavities which are suitable for analyte. Thus, a double imprinting approach enable us to tune the cavity size, because it can be structured by a small and a large template molecule than only by one type. The large compound acts as porogen and creates a better diffusion pathways to make the accessibility of the hollows much easier for the incoming analyte. 
Figure 7. Sensitivity of different double-imprinted layers to anthracene, exhibiting the influence of temperature and template ratios.

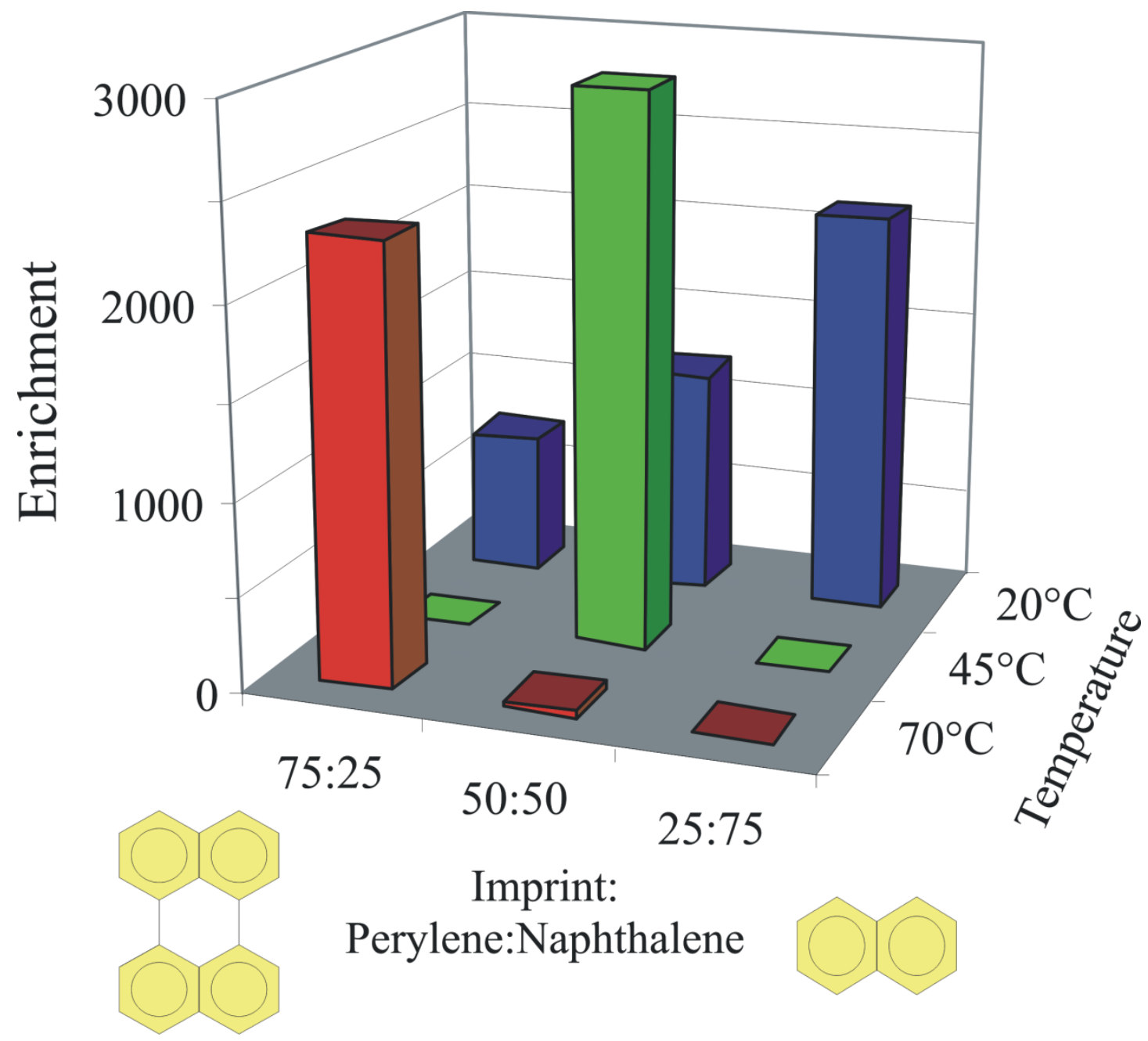

\subsubsection{Monitoring oil degradation}

Molecular imprinting as an advantageous technique allows us to generate a polymeric structure being capable of monitoring complex processes. Lubricating oil is a mixture of base material and additives, while, degradation of oil being very complex process affects the additives; then leads to the oxidation of base oil to form different acidic products. By following imprinting approach, a recognition system has been tuned in such a way that it can possibly characterize the chemical changes in engine oil and exhibit the complex oxidation process into a single sensor signal. An imprinted material templated with capric acid will generate such type of cavities, which can detect acidic components in used oil having five to fifteen carbon atoms.

The diffusion pathways along with the size of the cavities are very important for the inclusion of analytes. The flexibility of imprinting approach allows us to develop capric acid-templated nanoparticles of titania- and Zirconia. These nanoparticles offer substantially increased accessibility to the recognition sites for incoming analytes. Thus, a comparison of sensor effects of heat-treated Titania and Zirconia nanoparticles, while monitoring the degradation of engine oil is shown in figure 8 . The size of Titania nanoparticles is in the range of $50-100 \mathrm{~nm}$, while that of Zirconia is $100-200 \mathrm{~nm}$. 
Afterwards, these particles are heat-treated at 110, 220, 340, and $400^{\circ} \mathrm{C}$ and coated on dual electrode $10 \mathrm{MHz}$ QCM, while using non-imprinted titania sol-gel for anchoring the particles on one electrode and as reference material on second electrode for differential measurements. The mass-sensitive measurements are carried out at $80^{\circ} \mathrm{C}$ while exposing the 40 hours used oil. The results exhibit that at lower temperature, the mass effects are higher for titania nanoparticles due to their smaller size, which ensures greater surface area and improved diffusion. However, at higher temperatures, the pronounced sensor response is observed in case of zirconia particles, which are attributed to the enhanced stability of zirconia at high temperature.

Figure 8. Sensor effects of heat treated imprinted nanoparticles towards 40 hours used oil.

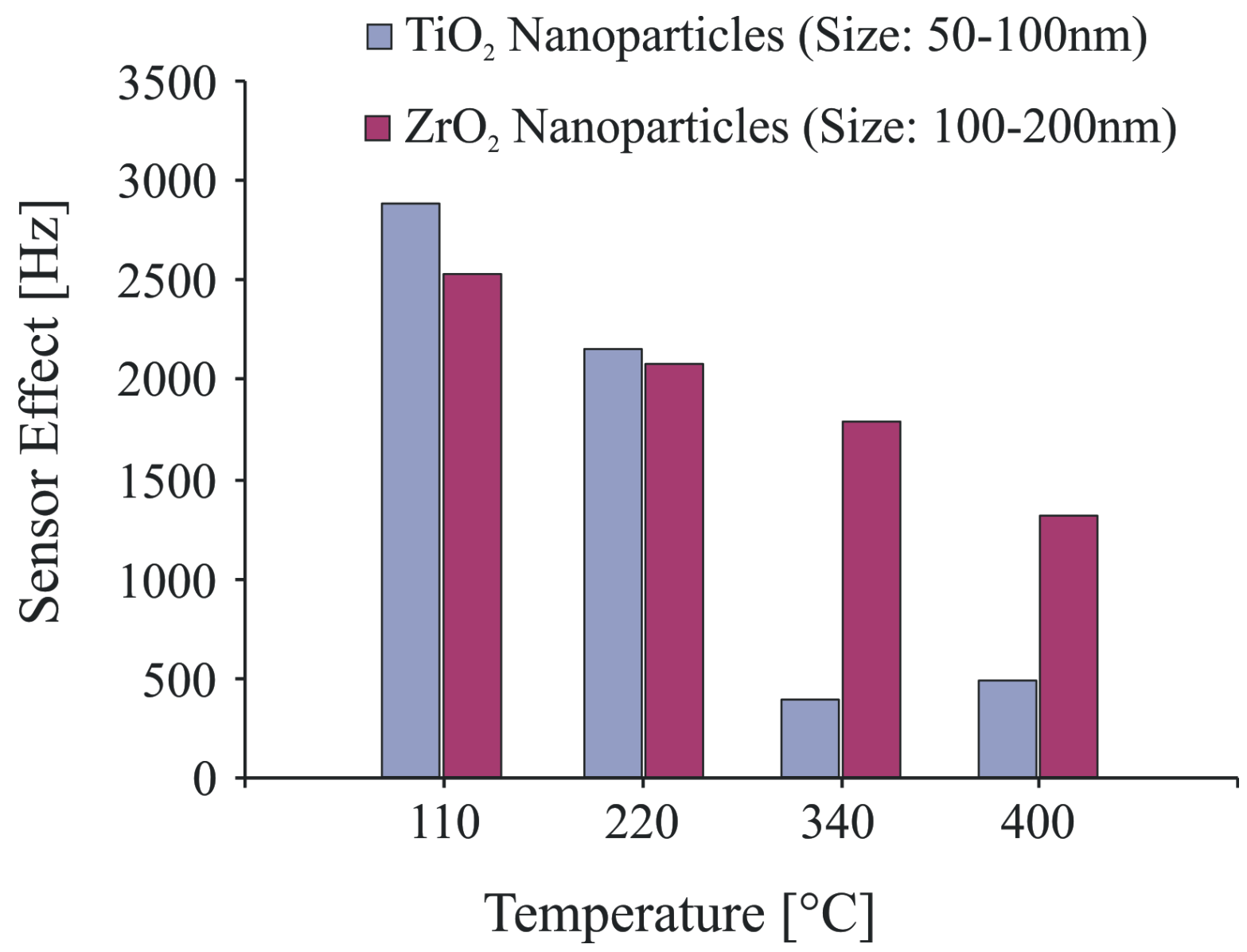

\subsubsection{Monitoring of composting process}

Molecular imprinting approach also help us to design a sensor array for online monitoring of complex processes i.e. composting procedures. To analyze the advancement in degradation process of plants, a sensor array is placed at headspace in the composter, where it can detect the complex analyte mixture emanate during composting. An array is fabricated by coating imprinted layers with different selectivities on mass-sensitive sensors being able to characterize the composting phases. For online monitoring in the composter, seven molecular imprinted polymers plus a non-imprinted polymer for differential measurements are coated on a combination of two $10 \mathrm{MHz}$ QCM (four electrode) to develop a sensor array. 
Figure 9. Selectivity profile of sensor array to 50ppm concentrations of each analyte.

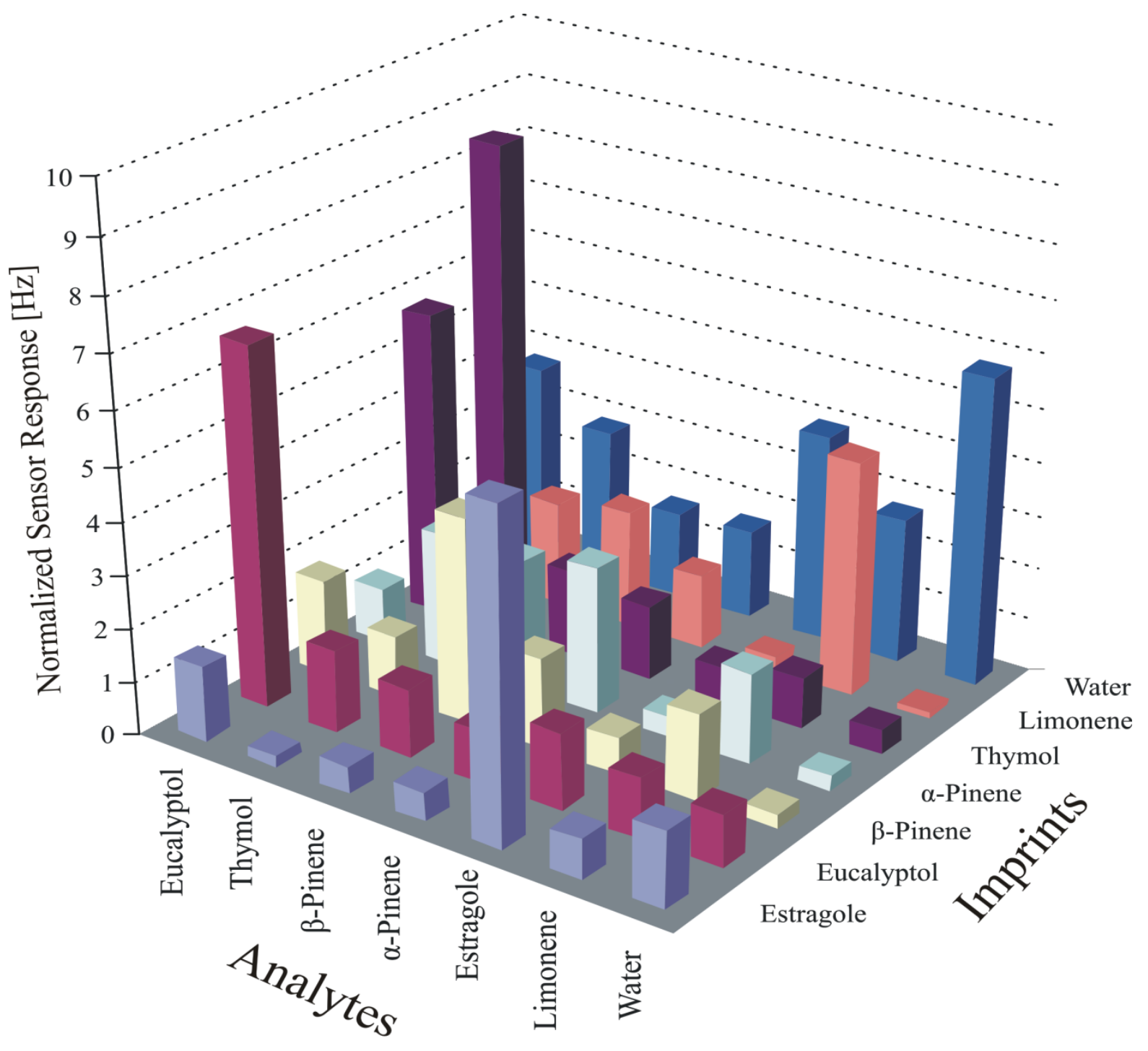

These imprinted polymers are tuned to detect water, limonene, estragole, $\alpha$-pinene, $\beta$-pinene, thymol, and eucalyptol which are the main compounds to characterize different degradation stages of herbs during composting. Polystyrene polymers are synthesized by templating the targeted analytes and the efficiency of device is analyzed by cross-sensitivities of eight sensor channels while exposing to 50ppm concentrations of each analyte. These effects are normalized according to layer height in order to compare the data, as shown in figure 9.

From these measurements, we can conclude that sensitive coatings show maximum selectivity towards their templated analyte. The figure also shows the capability of sensor array as it can easily differentiate between isomeric compounds such as $\alpha$-pinene and $\beta$-pinene. Thus, the designed sensor array proved to be a promising candidate for the online monitoring of composting. In the next step, the developed sensor system is equipped at the headspace of the commercially available composter for the online monitoring of degradation process of plants. The sensor array is also heated by a coil to avoid the humidity condensation on quartz surfaces and the data set for each analytes is represented in figure 10, while measuring the composting process up to 48 hours. 
Figure 10. Results based on QCM data - emanation of herbs as function of time in ppm.

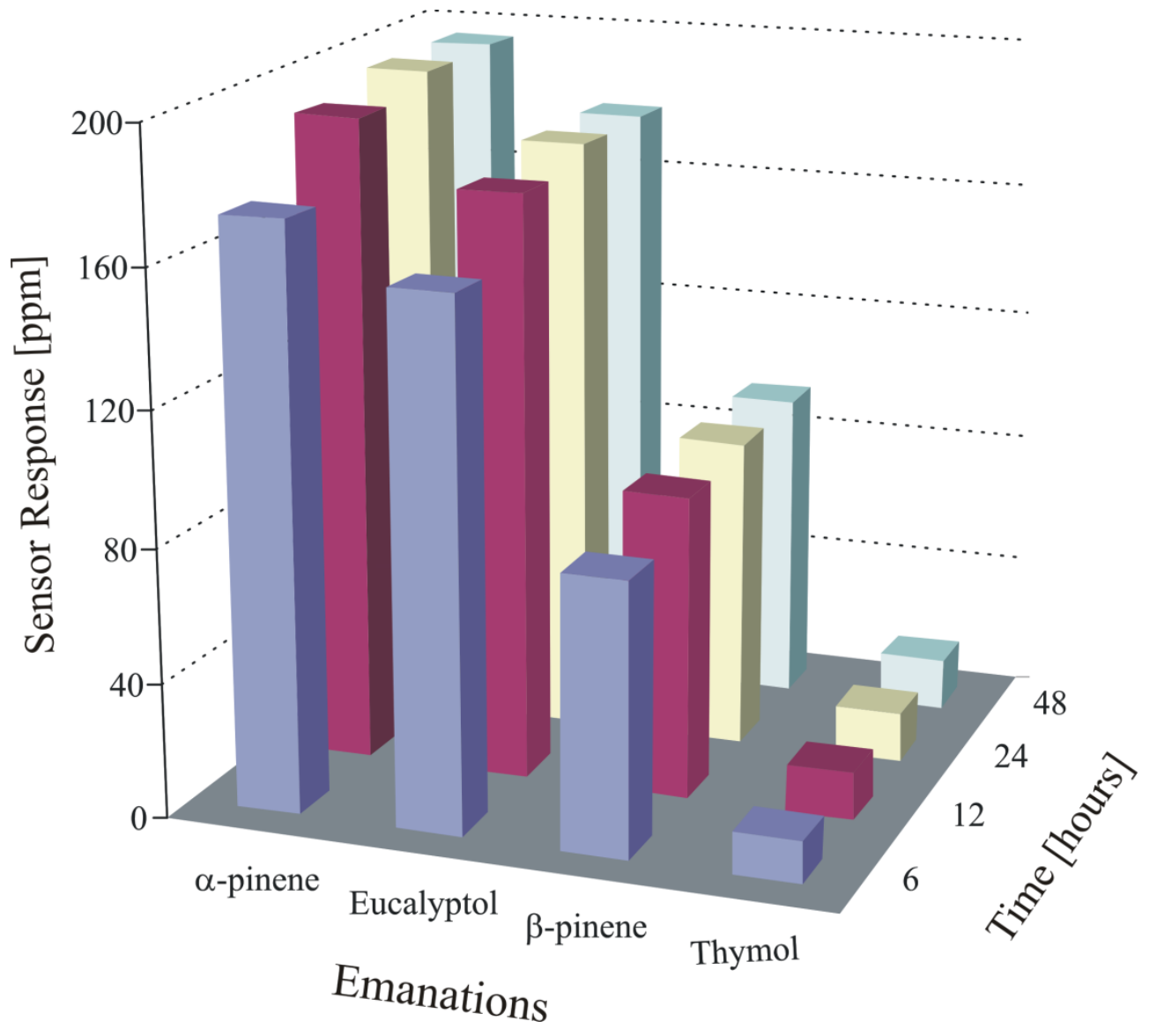

The result exhibit that four compounds emanate from herbs, whose concentration increases with time, as measured in terms of mass effects on QCM. Thus, the developed miniaturized sensor array based e-nose proved to be an artificial receptor like human olfactory system, which can recognize the emanating terpenes from herbs, even at the isomeric level.

\section{Experimental Section}

\subsection{Cyclodextrines}

$\alpha$-The $\beta$-cyclodextrines are obtained from Merck modified with hexafluorobenzene. Alkylation is carried out with alkyl iodides using $\mathrm{NaH}$ for deprotonation and silylation by following the procedure (30).

\subsection{Paracyclophanes}

The sensitive layer of paracyclophanes is synthesized as follows: At first, $N, N^{\prime}$-ditosylbenzidine is prepared by reacting toluene- $p$-sulfonic acid with benzidine $(2: 1)$ in pyridine and then, with $\mathrm{HCl}$. Afterwards, 1,4-dibromobutane is added along with DMF to produce a linkage to form bis$N, N^{\prime}$-ditosyl- $N, N^{\prime}$-tetramethylene-benzidine followed by precipitation and purification with water 
and chloroform, respectively. Moreover, tosyl units are eliminated by reacting with $\mathrm{HBr}$ and phenol to synthesize different derivatives followed by neutralization with $\mathrm{NaOH}$.

\subsection{Calixarenes}

Tert-butylcalix[4]arene and tert-butylcalix[8]arene are obtained from Sigma Aldrich. Tertbutylcalix[6]arene is synthesized by following standard procedure (31). The pertrimethylsilylation of calixarenes are carried out by using $N, O$ bis(trimethylsilyl)acetamide as silylating agent.

\subsection{Imprinted polyurethane for polycyclic aromatic hydrocarbons}

PAH-imprinted polyurethanes are prepared by dissolving $1 \mathrm{~g}$ of $\mathrm{p}, \mathrm{p}$-diisocyanato diphenylmethane (DPDI), $1.97 \mathrm{~g}$ of bisphenol $\mathrm{A}$ (BPA), and $0.22 \mathrm{~g}$ of phloroglucinol in tetrahydrofurane (THF) along with PAH templates (5\% of the mass of the monomers). Afterwards, polymerization is carried out at ambient conditions followed by heating between $20^{\circ} \mathrm{C}$ and $70^{\circ} \mathrm{C}$.

\subsection{Imprinted Titania and Zirconia nanoparticles}

Titania sol-gel is synthesized by dissolving $67 \mu \mathrm{L}$ of $\mathrm{Ti}(\mathrm{OBu})_{4}$ in $970 \mu \mathrm{L}$ of iso-propanol, while using $\mathrm{TiCl}_{4}$ as catalyst followed by polymerization at $60^{\circ} \mathrm{C}$ for $1 \mathrm{~h}$. Carbonic acid imprinted titania nanoparticles are prepared by dissolving $1040 \mathrm{mg}$ of capric acid in $6.5 \mathrm{~mL}$ of $\mathrm{CCl}_{4}$. Then, $178 \mu \mathrm{L}$ of $\mathrm{TiCl}_{4}$ is added and prehydrolyzed the solution at $60^{\circ} \mathrm{C}$ for half an hour. Afterwards, $63 \mu \mathrm{L}$ of water is inserted into solution drop wise while stirring, which leads to the precipitation of large sized particles. To obtain smaller sized nanoparticles, water is replaced by $100 \mu \mathrm{L}$ of $1 \mathrm{M}$ ammonium hydroxide. The nanoparticles are separated from solvent by centrifugation and for homogeneous size distribution; particles are suspended in $0.1 \mathrm{M} \mathrm{HCl}$ and sonicated for 5 minutes. At the end, particles are washed with water and acetone, respectively and then dried in ambient conditions.

Imprinted $\mathrm{ZrO}_{2}$ nanoparticles are generated by hydrolyzing the solution of zirconium propoxide with ammonium hydroxide. Zirconium propoxide $(327 \mathrm{mg}$ ) is dissolved in $5 \mathrm{~mL}$ of iso-propanol to form solution (A). Template solution (B) is synthesized by adding $172 \mathrm{mg}$ of template i.e. capric acid in $5 \mathrm{~mL}$ of iso-propanol. Afterwards, both the solutions (A \& B) are mixed and sonicated for half an hour. To precipitate the nanoparticles, ammonium hydroxide is added drop wise while stirring the solution. The solution is centrifuged to separate the particles, and then, washed with water and acetone followed by drying in air.

For anchoring on quartz electrodes, the nanoparticles are mixed with non-imprinted titania sol-gel layer, which act as a gluing material. The sizes of these nanoparticles are confirmed by using atomic force microscope in contact mode.

\subsection{Designing a sensor array}

The sensor array is designed by combining two quartz sheets, each containing four electrodes developed by screen printing via gold paste. Seven imprinted polystyrene polymers are generated by mixing $30 \mu \mathrm{L}$ of styrene functional monomer with $65 \mu \mathrm{L}$ of divinylbenzene as cross linker, while using AIBN (1mg) as radical initiator and $5 \mu \mathrm{L}$ diphenylmethane as porogen, followed by templating with targeted analytes. Afterwards, the mixture is polymerized at $70^{\circ} \mathrm{C}$ for 40 minutes to generate 
imprinted polymers. The sensitive layers of templated polymers are coated on the gold electrodes of sensor array by spinning at $2000 \mathrm{rpm}$. These layers are dried at room temperature and template will also evaporate leaving behind the cavities.

\subsection{Measurements}

The measurements are performed by using AT-cut quartz crystal microbalance (QCM) with a fundamental frequency of $10 \mathrm{MHz}$. Sensitivity of the system is further enhanced by adapting surface acoustic wave (SAW) device with frequency of $433 \mathrm{MHz}$, as mass sensitive sensor. The temperature and pressure fluctuations are eliminated by using an uncoated reference channel in each setup. The frequency of the sensor is measured by using a customized oscillator circuit combined with HP 53131A frequency counter and data acquisition is performed by LabVIEW via GPIB interface. Mass flow controllers (Tylan-RO7020) are used to allow the amounts of analyte vapors so as to generate required concentrations and humidity is measured by hygroscope (Rotronic DV-2). The HyperChem 5.0 is used for molecular modeling and force field calculations are executed by MM3 of Allinger (32). The fluorescence measurements are carried out with fluorescence spectrometer of Perkin-Elmer LS50B. The sensitive layer is coated on the quartz sheet in a thickness of $1.2 \mathrm{~mm}$, and excitation are perpendicular to sensor coatings, while emissions are observed at narrow face of the planar waveguide.

\section{Conclusions}

Cyclodextrine as host can successfully incorporate solvent vapors into the cavities. Moreover, the sensitivity of cyclodextrine can be tuned in such a way to differentiate between isomers. The supramolecular hollows offer suitable interactions to different solvent vapors but a more detailed understanding of incorporation is obtained by chemical modeling of host-guest complexes based on force field calculations. Molecular flexibility of calixarenes via conformational changes allows us to tune the recognition behavior according to guest molecule. However, another flexible technique, molecular imprinting, is more easily to be adapted to analytes without going through complex synthetic efforts. Moreover, double imprinting approach is used to tune the cavity size and suitable diffusion pathways for better accessibility of analytes. Molecular imprinting as an advantageous technique allows us to generate a polymeric structure being capable of monitoring complex processes, such as quality of engine oil or composting process. Titania- and Zirconia imprinted nanoparticles are applied as sensor coatings for monitoring the degradation of engine oil. At lower temperature, titania exhibit greater mass effects, whereas, pronounced responses are observed in case of Zirconia due to their enhanced stability at high temperature. A sensor array has also been developed by using imprinted polystyrene as sensitive coatings which can easily characterize the composting procedure.

\section{Acknowledgements}

We would like to acknowledge Higher Education Commission (HEC) of Pakistan for providing funding for $\mathrm{PhD}$ studies to Usman Latif. 


\section{References}

1. Pang W, Zhao H, Kim ES, Zhang H, Yu H, Hu X. 2012 Piezoelectric microelectromechanical resonant sensors for chemical and biological detection. Lab on a Chip - Miniaturisation for Chemistry and Biology; 12(1): 29-44.

2. Ramakrishnan N, Nemade HB, Palathinkal RP. 2012 Investigation on resonance effects of closely resonating nano-pillars attached to SAW resonator. Advanced Materials Research; Vol. 403-408, 1183-1187.

3. Yaqub S, Latif U, Dickert FL. 2011 Plastic antibodies as chemical sensor material for atrazine detection. Sensors and Actuators, B: Chemical; 160(1): 227-233.

4. Özalp VC. 2011 Acoustic quantification of ATP using a quartz crystal microbalance with dissipation. Analyst; 136(23): 5046-5050.

5. Sun P, Jiang YD, Xie GZ, Du XS, Li X, Hu J. 2011 Layer-by-layer assembly carbon nanotubes thin film based gas sensors for ammonia detection. Science China Information Sciences; 54(12): 2680-2686.

6. Lépinay S, Laffont G, Volet G, Wintgens V, Ferdinand P, Millot MC, Carbonnier B. 2012 Cyclodextrin-based supramolecular multilayer assemblies for the design of biological optical sensors using tilted fiber Bragg gratings. Key Engineering Materilas; Vol. 495, 45-48.

7. Hayden O, Latif U, Dickert FL. 2011 A mass-sensitive approach for the detection of anaesthetic xenon. Australian Journal of Chemistry; 64(12): 1628-1632.

8. Xu X, Liu Z, Zhang X, Duan S, Xu S, Zhou C. $2011 \beta$-Cyclodextrin functionalized mesoporous silica for electrochemical selective sensor: Simultaneous determination of nitrophenol isomers. Electrochimica Acta; 58(1): 142-149.

9. Dickert FL, Reif M, Sikorski R. 2001 Shaping tetraazaparacyclophanes for selective solvent vapour detection: Structural variations in coating design. Journal of Inclusion Phenomena; 40(12): 45-49.

10. Dickert FL, Geiger U, Weber K. 1999 Modified cyclodextrines as mass-sensitive coatings for solvent vapour detection. Fresenius J. Anal. Chem; 364(1-2): 128-132.

11. Dickert FL, Haunschild A, Reif M, Bulst W-E. 1993 Sensors for organic solvent detection in the ppm range based on dioxo[6.1.6.1]paracyclophanes-from molecular modeling to analyte recognition. Adv. Mater; 5 (4): 277-279.

12. Dickert FL, Schuster O. 1993 Mass sensitive detection of solvent vapors with calix[n]arenesconformational adaptation to the analyte. Adv. Mater; 5(11): 826-829.

13. Mokhtari B, Pourabdollah K. 2011 Structure optimization of di-ionizable calixarene nano-baskets for competitive solvent extraction of alkali and alkaline earth metals. Bulletin of the Korean Chemical Society; 32(11): 3855-3860.

14. Böhmer V. 1995 Calixarenes, macrocycles with (almost) unlimited possibilities. Angewandte Chemie (International Edition in English); 34(7): 713-745.

15. Hudrlik PF, Hudrlik AM, Arasho WD, Butcher RJ. 2008 A convenient preparation of C-silylated calixarenes. Synthesis; (18): 2968-2976.

16. Tabushi I. 1982 Cyclodextrin catalysis as a model for enzyme action. Acc. Chem. Res; 15(3): 6672. 
17. Gruber T, Fischer C, Seichter W, Bombicz P, Weber E. 2011 Upper rim site lipophilic calix[4]arenes as receptors for natural terpenes and functionally related solvent molecules: Combined crystal structure and QMB sensor study. Cryst Eng Comm; 13(5): 1422-1431.

18. Leila N, Sakina H, Abdelaziz B, Fatiha M, Fateh LLD. 2011 Theoretical study of the inclusion processes of the phenylurea herbicide metobromuron in $\beta$-cyclodextrin. Journal of Biological Sciences; 11(1): 1-9.

19. Al-Sou'od KA. 2006 Molecular mechanics study of the inclusion complexes of some 1,2,4oxadiazole derivatives of 3,3'-bis (1,2,4-oxadiazol-5(4H)-one) with $\beta$-cyclodextrin. Journal of Inclusion Phenomena; 54(1-2): 123-127.

20. El-Barghouthi MI, Al-Degs YS. 2004 Interactions of some organic compounds with $\alpha-$, $\beta$ - and $\gamma$ cyclodextrins: A molecular mechanics study. Asian Journal of Chemistry; 16(1): 207-212.

21. Yuan L, Ma J, Ding M, Wang S, Wu X, Li Y, Ma K, Zhou X, Li F. 2012 Preparation of estriolmolecularly imprinted silica nanoparticles for determining oestrogens in milk tablets. Food Chemistry; 131(3): 1063-1068.

22. Fu Y, Chen Z, Yu H, Yue Y, Di D. 2012 Preparation and adsorption selectivity of rutin molecularly imprinted polymers. Journal of Applied Polymer Science; 123(2): 903-912.

23. Ren Y, Ma W, Ma J, Wen Q, Wang J, Zhao F. 2012 Synthesis and properties of bisphenol A molecular imprinted particle for selective recognition of BPA from water. Journal of Colloid and Interface Science; 367(1): 355-361.

24. Latif U, Rohrer A, Lieberzeit PA, Dickert FL. 2011 QCM gas phase detection with ceramic materials - VOCs and oil vapors. Anal. Bioanal. Chem; 400(8): 2457-2462.

25. Schirhagl R, Latif U, Dickert FL. 2011 Atrazine detection based on antibody replicas. J. Mater. Chem; 21(38): 14594-14598.

26. Polreichova M, Latif U, Dickert FL. 2011 Functionalized polymers as receptors for detection of cells. Aust. J. Chem; 64(9): 1256-1260.

27. Dickert FL, Landgraf S, Sikorski R. 2000 Host-guest chemistry and chemical sensors: FT-IRAnalysis and spectra simulation of $\mathrm{CDCl}_{3}$ inclusion. J. Mol. Model; 6(6): 491-497.

28. Dickert FL, Reif M, Sikorski R, 2000 Chemical Sensors for Solvent Vapors: Enthalpic and Entropic Contributions to Host-Guest Interactions. Journal of Molecular Modeling; 6 (5) 446-451.

29. Dickert FL, Hayden O, 1999 Imprinting with sensor development - On the way to synthetic antibodies. Fresenius' Journal of Analytical Chemistry; 364 (6) 506-511.

30. Teranishi K, Ueno F. 2002 Regioselective Silylations of C-2 Hydroxyl Groups of Cyclodextrins Dependent on Reaction Temperature. Journal of Inclusion Phenomena and Macrocyclic Chemistry; 44(1): 307-312.

31. Vicens J, Bohmer V. 2004 Calixarenes: a versatile class of macrocyclic compounds. SpringerVerlag new york, ISBN: 0792307143

32. Allinger NL, Yuh YH, Lii JH. 1989 Molecular mechanics. The MM3 force field for hydrocarbons. Journal of the American Chemical Society; 111(23): 8551-8566.

(C) 2012 by the authors; licensee Insciences Journal.

Open Access article under the terms and conditions of Creative Commons Attribution Non-Commercial License 3.0 Unported. 